\title{
HARD THERMAL LOOPS, STATIC RESPONSE AND THE COMPOSITE EFFECTIVE ACTION*
}

\author{
R. Jackiw, Q. Liu, and C. Lucchesi ${ }^{\dagger}$ \\ Center for Theoretical Physics, Laboratory for Nuclear Science, and Department of Physics \\ Massachusetts Institute of Technology, Cambridge, Massachusetts 02139
}

\begin{abstract}
First, we investigate the static non-Abelian Kubo equation. We prove that it does not possess finite energy solutions; thereby we establish that gauge theories do not support hard thermal solitons. This general result is verified by a numerical solution of the equations. A similar argument shows that "static" instantons are absent. In addition, we note that the static equations reproduce the expected screening of the non-Abelian electric field by a gauge invariant Debye mass $m=g T \sqrt{\frac{N+N_{F} / 2}{3}}$. Second, we derive the non-Abelian Kubo equation from the composite effective action. This is achieved by showing that the requirement of stationarity of the composite effective action is equivalent, within a kinematical approximation scheme, to the condition of gauge invariance for the generating functional of hard thermal loops.
\end{abstract}

Submitted to: Physical Review D

CTP\#2261

November 1993

\footnotetext{
*This work is supported in part by funds provided by the U.S. Department of Energy (D.O.E.) under contract \#DE-AC02-76ER03069.

†Supported by the Swiss National Science Foundation.
} 


\section{INTRODUCTION}

When it was realized [1] that the gauge invariance condition [2] on the generating functional $\Gamma(A)$ for hard thermal loops in a gauge theory [3] (with or without fermions) coincides with a similar requirement on the wave functional of Chern-Simons theory, one could use the known solution for the latter, non-thermal problem [4] to give a construction of $\Gamma(A)$ relevant in the former, thermal context. The expression for $\Gamma(A)$ is non-local and not very explicit: $\Gamma(A)$ can be presented either as a power series in the gauge field $A$ [1] [the $O\left(A^{n}\right)$ contribution determines the hard thermal gauge field (and fermion) loop with $n$ external gauge field lines] or as an explicit functional of path ordered variables $P \exp \int d x^{\mu} A_{\mu}$ [四].

More accessible is the expression for the induced current $-\frac{\delta \Gamma(A)}{\delta A_{\mu}} \equiv-T^{a} \frac{\delta \Gamma(A)}{\delta A_{\mu}^{a}}$, which enters (high-temperature) response theory, in a non-Abelian generalization of Kubo's formula (in Minkowski space-time) [5]:

$$
D_{\nu} F^{\nu \mu}(x)=-\frac{\delta \Gamma(A)}{\delta A_{\mu}(x)} \equiv \frac{m^{2}}{2} j^{\mu}(x) .
$$

$T^{a}$ is an anti-hermitian representation of the Lie algebra, the gauge covariant derivative is defined as $D_{\nu}=\partial_{\nu}+g\left[A_{\nu},\right]$, and $m$ is the Debye mass determined by the matter content: in an $S U(N)$ gauge theory at temperature $T$, with fermions in the representation $\mathcal{T}^{a}$, and $\operatorname{Tr}\left(\mathcal{T}^{a} \mathcal{T}^{b}\right)=-\frac{N_{F}}{2} \delta^{a b}$ where $N_{F}$ counts the number of flavors, the Debye mass satisfies

$$
m^{2}=\frac{g^{2} T^{2}}{3}\left(N+\frac{N_{F}}{2}\right)
$$

Henceforth, through Section [1], we scale the gauge coupling constant to unity. The functional form of $j^{\mu}$ can be given as [5]

$$
j^{\mu}(x)=\int \frac{d \hat{q}}{4 \pi}\left\{Q_{+}^{\mu}\left(a_{-}(x)-A_{-}(x)\right)+Q_{-}^{\mu}\left(a_{+}(x)-A_{+}(x)\right)\right\} .
$$

Here $Q_{ \pm}^{\mu}$ are the light-like 4 -vectors $\frac{1}{\sqrt{2}}(1, \pm \hat{q})$, with $\hat{q}^{2}=1, A_{ \pm}$are the light-like projections $A_{ \pm}=Q_{ \pm}^{\mu} A_{\mu}$, while $a_{ \pm}$are given by [4, 5]

$$
a_{+}=g^{-1} \partial_{+} g, \quad a_{-}=h^{-1} \partial_{-} h \quad\left(\partial_{ \pm} \equiv Q_{ \pm}^{\mu} \partial_{\mu}\right)
$$


when $A_{ \pm}$are parametrized as

$$
A_{+}=h^{-1} \partial_{+} h, \quad A_{-}=g^{-1} \partial_{-} g .
$$

In other words, $a_{ \pm}$satisfy the equations

$$
\begin{aligned}
& \partial_{+} a_{-}-\partial_{-} A_{+}+\left[A_{+}, a_{-}\right]=0, \\
& \partial_{+} A_{-}-\partial_{-} a_{+}+\left[a_{+}, A_{-}\right]=0,
\end{aligned}
$$

whose solution can be presented as in (1.4) when $A_{ \pm}$are parameterized as in (1.5) - evidently $g$ and $h$ involve path ordered exponential integrals of $A_{ \pm}$. (Alternatively $a_{ \pm}$may be given by a power series in $A_{\mp}[1]$.) Finally (11.3) requires averaging over the directions of $\hat{q}$.

It is easy to verify that (1.6) ensure covariant conservation of $j^{\mu}$. Moreover, gauge invariance is maintained: for (11.1) to be gauge covariant, it is necessary that $j^{\mu}$ transform gauge covariantly. That the expression in (1.3) possesses this property is seen as follows. When $A_{ \pm}$transform by $U^{-1} A_{ \pm} U+U^{-1} \partial_{ \pm} U$, Eqs. (1.4) - (1.6) show that $a_{ \pm}$transform similarly, hence the differences $a_{ \pm}-A_{ \pm}$transform covariantly. The manifest gauge covariance of (1.1) ensures that $m$ is a gauge invariant parameter; that it also has the interpretation of an electric (Debye) mass will be evident when we consider the static limit.

It is of obvious interest to discuss solutions of (1.1). In the Abelian, electrodynamical case this is easy to do, since (1.6) can be readily solved for $a_{ \pm}$, and the solutions of the linear problem are the well-known plasma waves [6]. The non-linear problem of finding nonAbelian plasma waves is much more formidable. Also, one inquires whether the non-linear equations support soliton solutions, and (after an appropriate continuation to imaginary time) instanton solutions. [The time-dependent equation (1.1) in Minkowski space-time must be supplemented with boundary conditions, which are determined by the physical context. For example, response theory requires retarded boundary conditions, which in fact preclude deriving (1.1) variationally [5]. Here we shall not be concerned with this issue.]

Our paper concerns the following two topics. In Section [1], we analyze (11.1) for static fields. It turns out that in the time-independent case (1.6) can be solved for $a_{ \pm}$and (1.1) is 
presented in closed form. We prove that the resulting equation does not possess finite-energy solutions, thereby establishing that gauge theories do not support hard thermal solitons. Also some negative conclusions about instantons are given. In Section III] we present an alternative derivation of (1.1), which relies on the composite effective action [7], and makes use of approximations recently developed in an analysis of hard thermal loops based on the Schwinger-Dyson equations [8]. The Appendix presents a numerical analysis of the solutions to equation (1.1) for $S U(2)$, which supports the general result in Section 【.

\section{STATIC RESPONSE}

When $A_{ \pm}$are time-independent, we seek solutions of (1.6) that are also time-independent. Acting on static fields, the derivatives $\partial_{ \pm}$become $\pm \frac{1}{\sqrt{2}} \hat{q} \cdot \nabla \equiv \pm \partial_{\tau}$, and (1.6) may be written as the equations

$$
\partial_{\tau} \mathcal{A}_{ \pm} \pm\left[A_{ \pm}, \mathcal{A}_{ \pm}\right]=0
$$

for the unknowns $\mathcal{A}_{ \pm} \equiv A_{ \pm}+a_{\mp}$. These are solved trivially by $\mathcal{A}_{ \pm}=0$, that is

$$
a_{\mp}=-A_{ \pm}
$$

This solution is also the one that is deduced from the perturbative series expression for $a_{ \pm}$, when restricted to static $A_{ \pm}$.

[A non trivial solution can be constructed with the help of representations similar to (1.5). Upon defining in the static case

$$
\begin{aligned}
& A_{+}=h_{0}^{-1} \partial_{\tau} h_{0} \\
& A_{-}=-g_{0}^{-1} \partial_{\tau} g_{0}
\end{aligned}
$$

( $h_{0}$ and $g_{0}$ involve path-ordered exponentials along the path $\mathbf{r}+\hat{q} \tau$ ), we find

$$
\begin{aligned}
& \mathcal{A}_{+}=h_{0}^{-1} I_{+} h_{0}, \\
& \mathcal{A}_{-}=g_{0}^{-1} I_{-} g_{0},
\end{aligned}
$$


where $I_{ \pm}$are arbitrary Lie algebra elements, independent of $\tau: \hat{q} \cdot \nabla I_{ \pm}=0$. Since these solutions involve the arbitrary quantities $I_{ \pm}$, and since they do not arise in the perturbative series, we do not consider them further and remain with the trivial solution (2.2), which corresponds to $I_{ \pm}=0$.]

From (2.2) it follows that the current for static fields is

$$
\begin{aligned}
j^{\mu}(\mathbf{r}) & =-\int \frac{d \hat{q}}{4 \pi}\left(Q_{+}^{\mu}+Q_{-}^{\mu}\right)\left(A_{+}(\mathbf{r})+A_{-}(\mathbf{r})\right) \\
& =-\int \frac{d \hat{q}}{4 \pi}\left(Q_{+}^{\mu}+Q_{-}^{\mu}\right)\left(Q_{+}^{\nu}+Q_{-}^{\nu}\right) A_{\nu}(\mathbf{r}) .
\end{aligned}
$$

With $\mathbf{Q}_{+}+\mathbf{Q}_{-}=0$ and $Q_{+}^{0}+Q_{-}^{0}=\sqrt{2}$, we compute $j^{\mu}=-2 \delta^{\mu 0} A_{0}$. The response equations (1.1) then become, in the static limit:

$$
\begin{aligned}
D_{i} E^{i}+m^{2} A_{0} & =0, \\
\epsilon^{i j k} D_{j} B^{k} & =\left[A_{0}, E^{i}\right],
\end{aligned}
$$

where $E^{i} \equiv F^{i 0}$ and $F^{i j} \equiv-\epsilon^{i j k} B^{k}$. Eqs. (2.4) give clear indication that $m$ plays the role of a gauge invariant, electric mass. The fact that the static current is linear in the vector potential implies the vanishing of hard thermal loops with more that two external gauge-field lines, and zero energy - a fact which can be checked from the relevant graphs.

Unfortunately, Eqs. (2.4) do not possess any finite energy solutions. This is established by a variant of the argument relevant to the $m^{2}=0$ case 9 ].

Consider the symmetric tensor

$$
\theta^{i j}=2 \operatorname{Tr}\left(E^{i} E^{j}+B^{i} B^{j}-\frac{\delta^{i j}}{2}\left(E^{2}+B^{2}+m^{2} A_{0}^{2}\right)\right) .
$$

Using (2.4) one verifies that for static fields $\partial_{j} \theta^{j i}=0$. Therefore

$$
\int d^{3} r \theta^{i i}=\int d^{3} r \partial_{j}\left(x^{i} \theta^{j i}\right)=\int d S^{j} x^{i} \theta^{j i}
$$

Moreover, the energy of a massive gauge field (with no mass for the spatial components) can be written as

$$
\mathcal{E}=\int d^{3} r\left\{-\operatorname{Tr}\left(E^{2}+B^{2}+\frac{1}{m^{2}}\left(D_{i} E^{i}\right)^{2}\right)+\operatorname{Tr}\left(m A_{0}+\frac{D_{i} E^{i}}{m}\right)^{2}\right\} .
$$


The second trace in the integrand enforces the constraint 2.4a). Consequently, on the constrained surface the energy is a sum of positive terms [10]:

$$
\mathcal{E}=\int d^{3} r\left\{-\operatorname{Tr}\left(E^{2}+B^{2}+m^{2} A_{0}^{2}\right)\right\}
$$

and $\mathbf{E}, \mathbf{B}$ and $A_{0}$ must decrease at large distances sufficiently rapidly so that each of them is square integrable. This in turn ensures that the surface integral at infinity in (2.6) vanishes, so that static solutions require

$$
\int d^{3} r \theta^{i i}=0
$$

On the other hand, from (2.5), we see that $\theta^{i i}$ is a sum of positive terms

$$
\theta^{i i}=-\operatorname{Tr}\left(E^{2}+B^{2}+3 m^{2} A_{0}^{2}\right)
$$

hence (2.8) imply the vanishing of $\mathbf{E}, \mathbf{B}$ and $A_{0}$.

The absence of finite energy static solutions can also be understood from the differential equations (2.4). Eq. (2.4a) possesses solutions for $A_{0}$ that are either exponentially increasing or decreasing at infinity. Rejecting the former removes the freedom of imposing further conditions at the origin, and necessarily the exponentially damped solution devolves into one that is singular (not integrable) at the origin; see the Appendix. (This situation can be contrasted with, e.g., the magnetic dyon solution [11], where absence of the mass term allows solutions for $A_{0}$ with unconstrained large- $r$ behavior, leaving the freedom to select the solution that is regular at the origin.)

A similar argument shows that there are no "static" instanton solutions. These would be solutions for which $t$ is replaced by $-i x_{4}, A_{0}$ by $i A_{4}$ and presumably one would seek solutions periodic in $x_{4}$ with period $\beta=\frac{1}{T}=\frac{1}{m} \sqrt{\frac{N+N_{F} / 2}{3}}$. An $x_{4}$-independent solution is necessarily periodic; it would satisfy (2.4) with $A_{4}$ replacing $A_{0}$ and opposite sign in the right side of (2.4b). But analysis similar to the above shows that finite-action solutions do not exist. 


\section{HARD THERMAL LOOPS FROM THE COMPOSITE EFFECTIVE ACTION}

In this Section, we present a derivation of the non-Abelian Kubo equation (1.1) based on the composite effective action of [7], a generalization of the usual effective action (obtained by coupling local sources to the fields) in which one additionally introduces bilocal sources. In the QCD case, the composite effective action is given by $S(A)+\Gamma_{\mathrm{c}}\left(A, G_{\phi}\right)$, where $G_{\phi}(x, y)$ are (undetermined) two-point functions, and the labels $\phi=A, \psi, \zeta$ denote either gluons, or fermions-antifermions, or ghosts-antighosts, respectively (in the end, ghosts play no dynamical role, beyond maintaining gauge covariance of the final result). $S(A)$ is the pure Yang-Mills action, and

$$
\begin{aligned}
\Gamma_{\mathrm{c}}\left(A, G_{\phi}\right)= & \frac{i}{2}\left(\operatorname{Tr} \ln G_{A}^{-1}+\operatorname{Tr} \mathcal{D}_{A}^{-1} G_{A}\right) \\
& -i\left(\operatorname{Tr} \ln G_{\psi}^{-1}+\operatorname{Tr} \mathcal{D}_{\psi}^{-1} G_{\psi}+\operatorname{Tr} \ln G_{\zeta}^{-1}+\operatorname{Tr} \mathcal{D}_{\zeta}^{-1} G_{\zeta}\right)
\end{aligned}
$$

when 2PI contributions are omitted. The trace is over space-time arguments as well as over Lorentz and group indices. The gauge coupling constant $g$, which was previously scaled to

unity, is here reinserted. $\mathcal{D}_{\phi}^{-1}$ is computed from the usual QCD action $S_{Q C D}$ (e.g. in the Feynman gauge):

$$
i \mathcal{D}_{\phi}^{-1}(x, y)=\frac{\delta^{2} S_{Q C D}}{\delta \phi(x) \delta \phi(y)}
$$

The fields carry group and space-time indices, which are symbolically subsumed into the space-time labels $x, y$.

The truncated composite effective action (3.1) comprises the first, dynamical approximation that we make and reflects the known fact [3] that hard thermal loops arise from one-loop graphs. The full composite effective action of course coincides with the ordinary effective action when the two-point functions are evaluated by imposing stationarity requirements, and the above truncation reproduces the standard one-loop action involving $\operatorname{Tr} \ln \mathcal{D}_{\phi}^{-1}$. Nevertheless subsequent analysis is more transparently organized in the composite effective action formalism. 
As indicated in [7], $S+\Gamma_{\mathrm{c}}$ is stationary for physical processes. This yields the conditions

$$
\begin{aligned}
D_{\nu} F^{\nu \mu} & =J^{\mu}, \\
G_{\phi}^{-1} & =\mathcal{D}_{\phi}^{-1}, \quad \phi=A, \psi, \zeta .
\end{aligned}
$$

Computing the local induced current $J^{\mu}(x)=-\frac{\delta \Gamma_{\mathrm{c}}}{\delta A_{\mu}(x)}$ involves differentiating $\mathcal{D}_{\phi}^{-1}$ with respect to $A_{\mu}$. Since the $\mathcal{D}_{\phi}^{-1}$ depend locally on $A$, the resulting current is the local limit of a bilocal expression constructed from the two-point functions $G_{\phi}(x, y)$ :

$$
J^{\mu}(x)=\lim _{y \rightarrow x} J^{\mu}(x, y),
$$

where the bilocal current $J^{\mu}(x, y)=T^{a} J_{a}^{\mu}(x, y)$ is given by

$$
J^{\mu}(x, y)=g\left(\Gamma_{\alpha \beta \gamma}^{\mu} D_{x}^{\alpha} \mathbf{G}_{A}^{\beta \gamma}(x, y)+\partial_{y}^{\mu} \mathbf{G}_{\zeta}(x, y)\right)+i g T^{a} \operatorname{tr} \gamma^{\mu} \mathcal{T}^{a} G_{\psi}(x, y)
$$

with $\Gamma_{\alpha \beta \gamma}^{\mu} \equiv 2 g_{\beta}^{\mu} g_{\alpha \gamma}-g_{\alpha}^{\mu} g_{\beta \gamma}-g_{\gamma}^{\mu} g_{\beta \alpha}$. The trace "tr" is taken over Dirac spinor as well as internal symmetry indices, and we have defined $\mathbf{G}_{A, \zeta}(x, y)=\left[T^{a}, T^{b}\right] G_{A, \zeta a b}(x, y)$ with $D_{x} \mathbf{G}_{A}(x, y)=\partial_{x} \mathbf{G}_{A}(x, y)+g\left[\left[A(x), T^{b}\right], T^{c}\right] G_{A b c}(x, y)$.

We now use eqs. (3.3) - (3.5) to study "soft" plasma excitations. "Soft" means that both the energy and the momentum carried by a particle are of order $g T$, for a coupling constant $g \ll 1$, while particles with energy or momentum of order $T$ are called hard (see e.g. [3]). The strategy is to solve the system of coupled equations (3.3), in order to derive from (3.5) the expression (1.3) for the local current $J^{\mu}$. We approximate eqs. (3.3) by expanding them in powers of $g$. The approximation scheme we use was first proposed in [8] for deriving hard thermal loops from the Schwinger-Dyson equations. It represents an essential step in that derivation. Earlier work on the QCD plasma (in which this approximation was not used) is reviewed in [12]. Following [8], we introduce relative and center of mass coordinates, $s=x-y$ and $X=\frac{1}{2}(x+y)$, respectively. In these new variables the partial derivatives carry different dependences on $g: \partial_{s} \sim T$ and $\partial_{X} \sim g T$. This comes from the fact that $\partial_{s}$ corresponds to hard loop momenta, whereas $\partial_{X}$ is related to soft external momenta. See [8] for a detailed account. 
Next, motivated by the expression (3.4)-(3.5) for the current, we expand $G_{\phi}$ in powers of $g$ :

$$
G_{\phi}=G_{\phi}^{(0)}+g G_{\phi}^{(1)}+g^{2} G_{\phi}^{(2)}+\ldots
$$

where $G_{\phi}^{(0)}$ is just the free propagator at temperature $T$ and $G_{\phi}^{(i)}, i \geq 1$ are determined by (3.3b). At leading order in $g$ (to which we restrict ourselves in the sequel), the bilocal current 3.5$)$ depends on $G_{\phi}^{(0)}$ and $G_{\phi}^{(1)}$ :

$$
\begin{aligned}
J_{a}^{\mu}(X, s)= & g^{2} f^{a b c}\left[\Gamma_{\alpha \beta \gamma}^{\mu}\left(\partial_{s}^{\alpha} G_{A b c}^{(1) \beta \gamma}(X, s)+f^{b d e} A_{d}^{\alpha}(X) G_{A e c}^{(0) \beta \gamma}(X, s)\right)-\partial_{s}^{\mu} G_{\zeta b c}^{(1)}(X, s)\right] \\
& +i g^{2} \operatorname{tr} \gamma^{\mu} \mathcal{T}^{a} G_{\psi}^{(1)}(X, s)+\delta J_{a}^{\mu}(X, s)
\end{aligned}
$$

where $G_{\phi}(X, s) \equiv G_{\phi}\left(X+\frac{s}{2}, X-\frac{s}{2}\right)$ [and similarly for $J(X, s)$. We have added the term $\delta J_{a}^{\mu}(X, s)$ in order to compensate for the loss of gauge covariance due to non-locality:

$$
\delta J_{a}^{\mu}(X, s)=g^{2} s \cdot A^{b}(X)\left[f^{a c e} f^{b c d}\left(3 \partial_{\nu}^{s} G_{A d e}^{(0) \mu \nu}(s)+\partial_{s}^{\mu} G_{\zeta}^{(0) d e}(s)\right)+i \operatorname{tr} \mathcal{T}^{b} \mathcal{T}^{a} \gamma^{\mu} G_{\psi}^{(0)}(s)\right]
$$

Note that this term vanishes in the local limit.

Now, we derive from (3.3b) a condition on $G_{\phi}^{(1)}$. [It turns out to be convenient to expand, instead of (3.3b), the equivalent equations $\mathcal{D}_{\phi}^{-1} G_{\phi}=G_{\phi} \mathcal{D}_{\phi}^{-1}=I$, in which we disregard temperature-independent contributions.] The $\mathcal{O}(g)$-condition does not fix $G_{\phi}^{(1)}$ uniquely; hence we need to go to $\mathcal{O}\left(g^{2}\right)$. The condition so obtained on $G_{\phi}^{(1)}$ can be used to derive a constraint on the bilocal current. The subsequent derivation of this constraint [eq. (3.13)] is similar to the one given in [8], to which we refer the reader for details. Momentum space is most convenient, i.e.

$$
G_{\phi}(X, k)=\int d^{4} s e^{i k \cdot s} G_{\phi}(X, s)
$$

the explicit forms for the thermal parts of the free propagators being (e.g. in Feynman gauge):

$$
\begin{aligned}
& G_{A a b}^{(0) \mu \nu}(k)=-2 \pi \delta^{a b} g^{\mu \nu} \delta\left(k^{2}\right) n_{B}\left(k_{0}\right), \\
& G_{\psi}^{(0) m n}(k)=-2 \pi \delta^{m n} \not k \delta\left(k^{2}\right) n_{F}\left(k_{0}\right), \\
& G_{\zeta}^{(0) a b}(k)=2 \pi \delta^{a b} \delta\left(k^{2}\right) n_{B}\left(k_{0}\right),
\end{aligned}
$$


where $n_{B, F}\left(k_{0}\right)=1 /\left(e^{\beta\left|k_{0}\right|} \mp 1\right)$ are the bosonic and fermionic probability distributions.

Similarly, for the bilocal current in momentum space one writes

$$
J^{\mu}(X, k)=\int d^{4} s e^{i k \cdot s} J^{\mu}(X, s) .
$$

In the limit $s \rightarrow 0$, or equivalently $y \rightarrow x$, where $X=x$,

$$
J^{\mu}(x)=J^{\mu}(X)=\int \frac{d^{4} k}{(2 \pi)^{4}} J^{\mu}(X, k) .
$$

The resulting constraint on the bilocal current is [8]:

$$
Q \cdot D_{X} J^{\mu}(X, k)=4 \pi g^{2} Q^{\mu} Q^{\rho} k_{0} F_{\rho 0} \delta\left(k^{2}\right) \frac{d}{d k_{0}}\left[N n_{B}\left(k_{0}\right)+N_{F} n_{F}\left(k_{0}\right)\right],
$$

where $Q^{\mu} \equiv \frac{k^{\mu}}{k_{0}}=(1, \mathbf{Q})$.

Our next task is to make contact between (3.13) and the gauge invariance condition for the generating functional of hard thermal loops. Our strategy consists in transforming (3.13) into two distinct conditions for positive and negative $k_{0}$ 's, and in taking advantage of the symmetry properties that arise. We first integrate the equation (3.13) over $|\mathbf{k}|$ and $k_{0} \geq 0$. Due to the $\delta\left(k^{2}\right)$ on the right side, the bilocal current is non-vanishing only when $k_{0}=|\mathbf{k}|$; hence $\mathbf{Q}$ can be replaced by a unit vector $\hat{q} \equiv \frac{\mathbf{k}}{|\mathbf{k}|}$. The integration thus yields:

$$
Q_{+} \cdot D_{X} \mathcal{J}_{+}^{\mu}(X, \hat{q})=-2 \sqrt{2} \pi^{3} m^{2} Q_{+}^{\mu} Q_{+}^{\rho} F_{\rho 0}
$$

where we have defined

$$
\mathcal{J}_{+}^{\mu}(X, \hat{q})=\int|\mathbf{k}|^{2} d|\mathbf{k}| \int_{0}^{\infty} d k_{0} J^{\mu}(X, k)
$$

Similarly, upon introducing

$$
\mathcal{J}_{-}^{\mu}(X, \hat{q})=\int|\mathbf{k}|^{2} d|\mathbf{k}| \int_{-\infty}^{0} d k_{0} J^{\mu}(X, k)
$$

the integration of (3.13) over $|\mathbf{k}|$ and $k_{0} \leq 0$ gives:

$$
Q_{-} \cdot D_{X} \mathcal{J}_{-}^{\mu}(X, \hat{q})=-2 \sqrt{2} \pi^{3} m^{2} Q_{-}^{\mu} Q_{-}^{\rho} F_{\rho 0}
$$

wherefrom one sees that $\mathcal{J}_{-}^{\mu}(X,-\hat{q})$ satisfies the same equation (3.14) as $\mathcal{J}_{+}^{\mu}(X, \hat{q})$. 
Now, using $\int d^{4} k=\int d \Omega|\mathbf{k}|^{2} d|\mathbf{k}| d k_{0}$, we rewrite the expression (3.12) for the local current as $J^{\mu}(X)=\int \frac{d \hat{q}}{(2 \pi)^{4}}\left[\mathcal{J}_{+}^{\mu}(X, \hat{q})+\mathcal{J}_{-}^{\mu}(X, \hat{q})\right]$. Here, $\hat{q}$ can be replaced by $-\hat{q}$ in each term of the integrand separately, since $\hat{q}$ spans the whole solid angle. Therefore, we can write

$$
J^{\mu}(X)=\int \frac{d \hat{q}}{(2 \pi)^{4}} \mathcal{J}^{\mu}(X, \hat{q})
$$

where $\mathcal{J}^{\mu}(X, \hat{q})$ is defined as

$$
\mathcal{J}^{\mu}(X, \hat{q}) \equiv \mathcal{J}_{+}^{\mu}(X, \hat{q})+\mathcal{J}_{-}^{\mu}(X,-\hat{q})
$$

and satisfies, as a consequence of (3.14) and (3.17),

$$
Q_{+} \cdot D_{X} \mathcal{J}^{\mu}(X, \hat{q})=-4 \sqrt{2} \pi^{3} m^{2} Q_{+}^{\mu} Q_{+}^{\rho} F_{\rho 0}
$$

From this, after decomposing

$$
\mathcal{J}^{\mu}(X, \hat{q})=\tilde{\mathcal{J}}^{\mu}(X, \hat{q})-4 \sqrt{2} \pi^{3} m^{2} Q_{+}^{\mu} A_{0}
$$

we get as our final condition on the bilocal current:

$$
Q_{+} \cdot D_{X} \tilde{\mathcal{J}}^{\mu}(X, \hat{q})=4 \sqrt{2} \pi^{3} m^{2} Q_{+}^{\mu} \partial_{X}^{0}\left(Q_{+} \cdot A\right)
$$

Let us now assume that $\tilde{\mathcal{J}}^{\mu}(X, \hat{q})$ can be obtained from a functional $W(A, \hat{q})$ as

$$
\tilde{\mathcal{J}}^{\mu}(X, \hat{q})=\frac{\delta W(A, \hat{q})}{\delta A_{\mu}(X)} .
$$

Equation (3.22) then implies that $W(A, \hat{q})$ depends only on $A_{+}$, i.e. $W(A, \hat{q})=W\left(A_{+}\right)$, and $\tilde{\mathcal{J}}^{\mu}=\frac{\delta W\left(A_{+}\right)}{\delta A_{+}} Q_{+}^{\mu}$. In turn, $W\left(A_{+}\right)$satisfies, as a consequence of (3.22),

$$
Q_{+} \cdot D_{X} \frac{\delta W\left(A_{+}\right)}{\delta A_{+}}=4 \sqrt{2} \pi^{3} m^{2} \partial_{X}^{0} A_{+} .
$$

By introducing new coordinates $\left(x_{+}, x_{-}, \mathbf{x}_{\perp}\right)$,

$$
x_{+}=Q_{-} \cdot X, \quad x_{-}=Q_{+} \cdot X, \quad \mathbf{x}_{\perp} \cdot \hat{q}=0
$$

we can rewrite $Q_{+} \cdot \partial_{X}$ as $\partial_{+}$and (3.24) becomes 


$$
\partial_{+} \frac{\delta W\left(A_{+}\right)}{\delta A_{+}}+g\left[A_{+}, \frac{\delta W\left(A_{+}\right)}{\delta A_{+}}\right]=4 \sqrt{2} \pi^{3} m^{2} \partial_{X}^{0} A_{+} .
$$

This equation was first derived in [2], as an expression of gauge invariance of the generating functional for hard thermal loops, and has since then been studied by several authors. Here, it is seen to be a consequence of the stationarity requirement on the composite effective action.

It has been shown in [1] that $W\left(A_{+}\right)$is given by the eikonal of a Chern-Simons gauge theory. This observation is our last step towards deriving the approximate expression for the local current $J^{\mu}(x)$ in eq. (3.3a). The subsequent development follows [5] and the result is exactly the non-Abelian Kubo equation (1.1) with the form (1.3) for the induced current.

\section{CONCLUSIONS}

The behavior of the quark-gluon plasma at high temperature is described by the nonAbelian Kubo equation (1.1) - (1.3). We have studied the static response of such a plasma and proved that there are no hard thermal solitons (this result is supported and illustrated by numerical integration). The absence of "static" instantons is established by invoking a similar argument. In addition, the static non-Abelian Kubo equation indicates that the non-Abelian electric field is screened by a gauge invariant Debye mass $m=g T \sqrt{\frac{N+N_{F} / 2}{3}}$.

Furthermore, we have derived the non-Abelian Kubo equation from the composite effective action formalism. Indeed, the requirement that the composite effective action be stationary leads, within a kinematical approximation scheme taken at the leading order, to the equation obtained in [2] by imposing gauge invariance on the generating funtional of hard thermal loops.

Let us mention some problems deserving further investigation. Finding non-static solutions to the non-Abelian Kubo equation is an appealing - if difficult - task, since such solutions would correspond to collective excitations of the quark-gluon plasma at high temperature. Also, it would be interesting to investigate the next-to-leading order effects in 
the kinematical approximation and to see if they are gauge invariant; we hope that our formalism is well suited for such an investigation. Furthermore, it is clear that $\Gamma_{\mathrm{c}}\left(A, G_{\phi}\right)$, when evaluated on the solution for $G_{\phi}$ obtained from (3.3b) and (3.6), coincides with the $\Gamma(A)$ constructed from the Chern-Simons eikonal. While our derivation establishes this fact indirectly, an explicit evaluation of the relevant functional determinants in the hard thermal limit would be welcome.

\section{NOTE ADDED}

We have now seen recent papers [13] wherein the response equations are also analyzed. Moreover, local equations are found for time-dependent, but space-independent gauge fields, and for non-Abelian plane waves. The starting point of these investigations is a non-local expression for the induced current (see 8,13$]$ ),

$$
j_{\mu a}^{\text {ind }}(x)=3 \omega_{p}^{2} \int \frac{d \Omega}{4 \pi} v_{\mu} \int_{0}^{\infty} d u U_{a b}(x, x-v u) \mathbf{v} \cdot \mathbf{E}^{b}(x-v u)
$$

which appears different from our local, but coupled, form $(1.3)-(1.6)$. Here we exhibit the steps that explicitly relate the two.

Beginning with our form for the induced current, (1.3) - (1.6), we observe that, owing to the integration over the angles of $\hat{q}$, we may collapse these expressions into

$$
\frac{m^{2}}{2} j^{\mu}(x)=m^{2} \int \frac{d \hat{q}}{4 \pi} Q_{+}^{\mu}\left(a_{-}(x)-A_{-}(x)\right)
$$

where

$$
\partial_{+} a_{-}+\left[A_{+}, a_{-}\right]=\partial_{-} A_{+}
$$

Eq. (3) may be integrated, yielding

$$
a_{-}^{a}(x)=\int_{0}^{\infty} d u U_{a b}\left(x, x-Q_{+} u\right) \partial_{-} A_{+}^{b}\left(x-Q_{+} u\right)
$$

Here $U_{a b}$ satisfies 


$$
\begin{aligned}
\frac{\partial}{\partial u} U_{a b}\left(x, x-Q_{+} u\right) & =U_{a c}\left(x, x-Q_{+} u\right) f_{c b d} A_{+}^{d}\left(x-Q_{+} u\right), \\
U_{a b}(x, x) & =\delta_{a b} .
\end{aligned}
$$

Also $A_{-}^{a}$ may be presented as

$$
\begin{gathered}
A_{-}^{a}(x)=-\int_{0}^{\infty} d u \frac{d}{d u}\left\{U_{a b}\left(x, x-Q_{+} u\right) A_{-}^{b}\left(x-Q_{+} u\right)\right\} \\
=\int_{0}^{\infty} d u U_{a b}\left(x, x-Q_{+} u\right)\left\{\partial_{+} A_{-}^{b}\left(x-Q_{+} u\right)\right. \\
\left.\quad-f^{b c d} A_{-}^{c}\left(x-Q_{+} u\right) A_{+}^{d}\left(x-Q_{+} u\right)\right\} .
\end{gathered}
$$

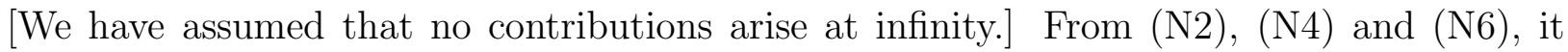
follows that the induced current can be written as

$$
\frac{m^{2}}{2} j_{a}^{\mu}(x)=m^{2} \int \frac{d \hat{q}}{4 \pi} Q_{+}^{\mu} \int_{0}^{\infty} d u U_{a b}\left(x, x-Q_{+} u\right) F_{-+}^{b}\left(x-Q_{+} u\right)
$$

which coincides with the expression (N1) derived in [13], after the notational replacements $m \rightarrow \sqrt{3} \omega_{p}, d \hat{q} \rightarrow d \Omega, Q_{+}^{\mu} \rightarrow v^{\mu}$ and $F_{-+} \rightarrow \mathbf{v} \cdot \mathbf{E}$ are performed.

The time-dependent, space-independent equation found in [13] is easily derived in our formalism, also. When there is no space dependence, eqs. (1.6) can be written as

$$
\partial_{+}\left(a_{\mp}-A_{ \pm}\right)+\left[A_{ \pm}, a_{\mp}-A_{ \pm}\right]=0
$$

and are solved by $a_{\mp}=A_{ \pm}$. Hence:

$$
\frac{m^{2}}{2} j^{\mu}=\frac{m^{2}}{2} \int \frac{d \hat{q}}{4 \pi}\left(Q_{+}-Q_{-}\right)^{\mu}\left(Q_{+}-Q_{-}\right)^{\nu} A_{\nu},
$$

of which only the spatial component is non-vanishing:

$$
\frac{m^{2}}{2} j^{i}=m^{2} \int \frac{d \hat{q}}{4 \pi} \hat{q}^{i} \hat{q}^{j} A_{j}=-\frac{1}{3} m^{2} A^{i} .
$$

This coincides with the result in [13].

Similarly, the induced current for the non-Abelian plane wave in [13 corresponds to:

$$
a_{ \pm}=\frac{Q_{ \pm} \cdot p}{Q_{\mp} \cdot p} A_{\mp}(p \cdot x)
$$


in our formalism, with $p=(\omega, \vec{k})$ being the corresponding wave vector.

\section{APPENDIX}

In this Appendix we analyze in greater detail and integrate numerically the radially symmetric version of the static response equations (2.4), in the $S U(2)$ case. Radially symmetric $S U(2)$ gauge potentials take the forms:

$$
\begin{aligned}
& A_{i}^{a}=\left(\delta^{a i}-\hat{r}^{a} \hat{r}^{i}\right) \frac{\phi_{2}(r)}{r}+\varepsilon^{a i j} \hat{r}^{j} \frac{1-\phi_{1}(r)}{r}, \\
& A_{0}^{a}=\hat{r}^{a} \frac{g(r)}{r},
\end{aligned}
$$

where a residual gauge freedom has been used to eliminate a term proportional to $\hat{r}^{a} \hat{r}^{i}$.

We substitute the Ansatz (A1) into (2.4). The resulting equations give us the freedom to set one of the two $\phi_{i}$ 's to zero; we obtain,

$$
\begin{aligned}
x^{2} \frac{d^{2}}{d x^{2}} J & =\left(x^{2}+2 K^{2}\right) J, \\
x^{2} \frac{d^{2}}{d x^{2}} K & =\left(K^{2}-J^{2}-1\right) K,
\end{aligned}
$$

where we have set $\phi_{2}$ to zero, rescaled $x=m r$ and defined $J(x)=g(r), K(x)=\phi_{1}(r)$.

We now investigate this system of coupled second-order differential equations. First, we see that they possess the following two exact solutions:

$$
\begin{aligned}
& J=0, K= \pm 1 \\
& J=J_{0} e^{-x}, K=0 .
\end{aligned}
$$

Eq. (A3a) corresponds to the Yang-Mills vacuum, while (A3b) is the celebrated Wu-Yang monopole plus a screened electric field.

In the asymptotic region $x \rightarrow \infty$, the regular solution of the system (A2) tends to (A3a), with $J$ approaching its asymptote exponentially. (Of course there is also the solution with $J$ growing exponentially, which we do not consider.) 
Near the origin, $J$ and $K$ behave either like the vacuum (A3a) or approach the monopole solution (A3b) as follows,

$$
\begin{aligned}
J(x) & \rightarrow J_{0}+\ldots, \\
K(x) & \rightarrow K_{0} \sqrt{x} \cos \left(\frac{2 \pi}{\tau} \ln \frac{x}{x_{0}}\right)+\ldots,
\end{aligned}
$$

where $\tau$ is correlated with $J_{0}$ as

$$
\tau=\frac{4 \pi}{\sqrt{4 J_{0}^{2}+3}} .
$$

Only the vacuum alternative at the origin leads to finite energy. However, since we must choose one of two possible solutions at infinity (obviously we pick the regular one), the behavior at the origin is determined and can be exhibited explicitly by integrating the equations (A2) numerically. Starting with regular boundary conditions at infinity, we find the profiles presented in Figure 1. They show that the monopole solution (A3B) is reached at the origin, with $K$ vanishing as in (A4) - (A5), a result consistent with our analytic proof that there are no finite energy static solutions in hard thermal gauge theories. 


\section{REFERENCES}

[1] R. Efraty and V. P. Nair, Phys. Rev. Lett. 68, 2891 (1992); Phys. Rev. D 47, 5601 (1993).

[2] J. C. Taylor and S. Wong, Nucl. Phys. B346, 115 (1990).

[3] E. Braaten and R. Pisarski, Phys. Rev. D 42, 2156 (1990), 45, 1827 (1992); Nucl. Phys. B337, 569 (1990), B339, 310 (1992).

[4] D. Gonzales and A. Redlich, Ann. Phys. (NY) 169, 104 (1986); G. Dunne, R. Jackiw and C. Trugenberger, Ann. Phys. (NY) 149, 197 (1989).

[5] R. Jackiw and V. P. Nair, Phys. Rev. D 48, 4991 (1993).

[6] V. P. Silin, Zh. Eksp. Teor. Fiz. 38, 1577 (1960) [Engl. trans: Sov. Phys. JETP 11, 1136 (1960)]; E. Lifshitz and L. Pitaevskii, Physical Kinetics (Pergamon, Oxford, 1981).

[7] J. M. Cornwall, R. Jackiw and E. Tomboulis, Phys. Rev. D 10, 2428 (1974).

[8] J. Blaizot and E. Iancu, Phys. Rev. Lett. 70, 3376 (1993); Saclay preprint SPhT/93-064.

[9] S. Deser, Phys. Lett. 64B, 463 (1976).

[10] That the energy is positive on the constrained surface even for non-static fields has been shown by V. P. Nair, Phys. Rev. D 48, 3432 (1993).

[11] B. Julia and A. Zee, Phys. Rev. D 11, 2227 (1975).

[12] H.-Th. Elze and U. Heinz, Phys. Rep. 183, 81 (1989).

[13] J. Blaizot and E. Iancu, Saclay preprints T94/02, T94/03, January 1994, and T94/013, February 1994. 